\title{
Gaspar Gómez de Toledo y la búsqueda de la fama
}

\author{
Luis M. Esteban Martín \\ Colegio Nuestra Señora de las Maravillas-La Salle (Madrid)
}

\section{RESUMEN}

Gaspar Gómez de Toledo, con su Tercera parte de la tragicomedia de Celestina, pretende no solo continuar la obra precedente, la Segunda Comedia de Celestina, de Feliciano de Silva, y entrar así en el ciclo celestinesco iniciado con la publicación de Celestina, sino convertirse en el autor con el que dicho ciclo quede cerrado definitivamente, de ahí que vaya concluyendo episodios inconclusos en la obra de Silva y que acabe definitivamente con la vieja alcahueta Celestina resucitada por Silva. Esta intención de cerrar un ciclo viene motivada por el deseo de fama, de pasar a la posteridad como el autor que culminó las aventuras de la alcahueta de Fernando de Rojas y, por tanto, gozar del éxito que el público otorgó a las dos obras que le precedieron.

Palabras clave: Gaspar Gómez de Toledo, Feliciano de Silva, ciclo celestinesco, fama, Fernando de Rojas

\section{Gaspar Gómez de Toledo and the pursuit of fame}

\section{ABSTRACT}

Gaspar Gómez de Toledo, with his Tercera parte de la tragicomedia de Celestina, intends not only to continue the previous work, the Segunda Comedia de Celestina, by Feliciano de Silva, and thus enter the celestinesque cycle that began with the publication of Celestina, but also to become the author with whom the cycle is definitively closed. Consequently he concludes unfinished episodes in Silva's work to definitively end with the old pimp Celestina resurrected by Silva. This intention to close a cycle is motivated by the desire for fame, in order to pass into posterity as the author who culminated the adventures of Fernando de Rojas' procurer and, therefore, to enjoy the success that the public granted to the two works that preceded him.

Keywords: Gaspar Gómez de Toledo, Feliciano de Silva, celestinesque cycle, fame, Fernando de Rojas 
A M.C., por tanto

En 1536, en Medina del Campo, aparecía la primera edición de la intitulada como Tercera parte de la tragicomedia de Celestina, de Gaspar Gómez, "natural de la muy insigne ciudad de Toledo», como se lee debajo de la xilografía y del título. En 1539 aparecería una segunda edición, en Toledo ${ }^{1}$. A estas dos ediciones Ticknor (1851: 282) añade una toledana de 1537 que, si bien Barrick (1973: 70) considera de existencia dudosa, me parece más bien un error por 1536, lo mismo que ocurre con la edición que Barrera data en Toledo en 1559 y que, esta vez sí de acuerdo con Ticknor, es una evidente errata por 1539.

La obra, de un autor del que nada sabemos y al que Urquijo alude como "oscuro escritor toledano» (1910: 574), pero que, sin embargo, posee una considerable cultura como se pone de manifiesto en las citas de autores griegos, latinos, eclesiásticos, mitología y literatura castellana ${ }^{2}$, aparece cuando Celestina tiene un éxito consolidado y además cuenta ya con una continuación de la mano de Feliciano de Silva, autor al que Gómez de Toledo dedica su obra.

En 1536, Celestina no solo cuenta con un buen elenco de ediciones, sino también de traducciones, antes de la publicación de la obra de Gaspar Gómez, como la que Alfonso Ordóñez hizo al italiano en 1506, la alemana de M. Wirsung en 1520, una al francés en 1527 que tuvo dos reimpresiones en 1529 y 1532 , y en 1530 se editó una traducción al inglés en verso de los cuatro primeros actos de la obra, probablemente, de J. Rastell.Pero no solo eso, sino que la obra firmada por Rojas era una auténtica obra popular incluso por encima de los libros de caballerías, como ha señalado Maxime Chevalier (1976: 146), puesto que circuló de manera oral o manuscrita de forma fragmentada. Y es tanta la popularidad de esta obra que, en el proceso por hereje y apóstata contra Álvaro de Montalván, en 1525, al hablar el acusado de su genealogía y llegar a sus hijos señala: «Leonor Aluares, mujer del Bachiller que compuso á Melibea, veçino de Talauera; aurá XXXV años» (Serrano Sanz 1902: 263).

Esta popularidad de Celestina, que abarca no solo a los círculos letrados, sino al público en general, teniendo en cuenta que, además, en la época lectores no solo son los que leen, sino aquellos a quienes se les lee, máxime si hablamos de comedias humanísticas, es la que anima a Feliciano de Silva, un noble ya conocido antes de 1536 por sus libros de caballlerías ${ }^{3}$,

1.- Ambas ediciones han sido descritas por Barrick (1973: 65-70) en su edición de la Tercera parte de la tragicomedia de Celestina. Para todas las citas de la Tercera Celestina sigo esta edición.

2.- Véase Esteban Martín (1986: 26-105).

3.- En 1514 se publicó el Lisuarte de Grecia (que contó con trece ediciones hasta 1578), en 1530 el Amadís de Grecia y entre 1532 y 1551 Don Florisel de Niquea o Rogel de Grecia. Es más, los comentarios que sus coetáneos hacen respecto a Silva como autor dan cuenta de la im- 
a publicar en 1534, en Medina del Campo, a cargo de Pedro Tovans, la Segunda Comedia de Celestina, obra que contó con otra edición en 1536, en Venecia, realizada por Stephano da Sabio, la de Salamanca, 1536, por Pedro de Castro, y la de Amberes, esta ya posterior a la obra de Gaspar Gómez de Toledo, de hacia 1540 o 1550 (Silva 1988: 91-92). Así pues, con Feliciano de Silva se abre lo que se ha dado en llamar el ciclo celestinesco ${ }^{4}$ y de saberse integrante de dicho ciclo da buena cuenta no sólo la obra de Gaspar Gómez de Toledo, sino que años después, Alonso de Villegas, en el prólogo de su Comedia Selvagia (1554: fol. iv), aluda a su osadía por tratar un tema que han tratado otros tan bien desarrollado, señalando explícitamente a Feliciano de Silva.

Pero lo más significativo, y que tiene relación con la actitud de Gaspar Gómez al afrontar su continuación de Celestina, es cómo Silva decide continuar el texto original, porque en ello confluyen una serie de elementos comunes al resto de continuadores. Abrir o integrarse en un ciclo literario está ligado al éxito editorial de los precedentes, como ha señalado Lázaro Carreter (1978: 196), y con ello, como señalaré más adelante, con la búsqueda de la fama entre los lectores; además, ese ser continuador supone el ser original con respecto al modelo y esto conlleva dos aspectos a tener en cuenta. Por un lado, que el continuador no es un mero imitador (Lázaro Carreter 1976: 117), sino que, como señala Gérard Genette, busca «una producción nueva: la de otro texto en el mismo estilo, de otro mensaje en el mismo código»(1989: 103), dentro del concepto renacentista de la imitatio y, más en concreto, siguiendo el tratado de Bartolomeo Ricci, De imitatione (1541) del concepto de aemulare, donde hay un reconocimiento del modelo del que se parte para destacar el reto que supone y también la novedad introducida (García Galiano 1992: 8). Por otro lado, y ligado con lo anterior, está el concepto de originalidad en el Renacimiento, que posibilita la utilización de temas, pensamientos, etc., de obras y autores precedentes (Pérez-Rioja 1988: 65), condicionado, obviamente, por el público al que se dirige la creación (Pérez-Rioja 1988: 108).

Así pues, Feliciano de Silva va a introducir en su continuación una serie de innovaciones como las cartas amorosas (Esteban Martín 2003), intensificará y modificará el papel de los criados y el mundo lupanario, planteará el matrimonio secreto como solución a los amores (recuérdese el rechazo explícito de Melibea al matrimonio en el auto XII de Celestina), incorporará pastores como Filínides, o los negros Zambrán y Boruca ${ }^{5}$. Pero, sobre todo, Silva tendrá una importancia capital para el resto de los

portancia de este escritor, tal y como ha recogido Consolación Baranda en su edición de la Segunda Celestina (1988: 28-30).

4.- Sobre la actuación como continuador de Feliciano de Silva véase Esteban Martín (1991-92).

5.- Una relación de todas las novedades incorporadas por Silva puede verse en la edición de la Segunda Celestina, de Consolación Baranda. 
continuadores por dos razones esenciales: recupera a la vieja alcahueta rojana y deja su obra abierta para posibles continuaciones.

Feliciano de Silva es consciente de lo que el público y editores comprendieron casi desde el instante en que aparece Celestina: la vieja alcahueta es el personaje más excepcional de la obra, con una configuración muy superior a la de antecedentes como Trotaconventos del arcipreste de Hita. Es la vieja Celestina la que desde su entrada en la obra se adueña de todos y cada uno de los momentos esenciales de la trama, hasta el punto de que en 1511, como ha señalado Kirby (1989: 60), en el testamento e inventario de bienes de Francisco de Treviño, regidor de Santiago de Compostela, se lee que entre sus libros figuraba "otro de celestina», lo que supone un ejemplo evidente de la popularidad de la vieja alcahueta, que, con posterioridad, se incorporará al título de la obra en la reimpresión hecha en 1519 de la versión italiana de Ordóñez y en la impresión del Libro de Calixto y Melibea $y$ de la puta vieja Celestina, fechado en Sevilla, en 1502; si bien, como ha anotado Norton, esta edición, así como todas las que aparecen en Sevilla o Toledo, en 1502, en realidad fueron publicadas entre 1510 y 1529, y ésta en concreto hacia 1518-20 (Norton 1966: 141-156). Y una prueba más de la importancia que se le concederá a la vieja Celestina es que, una vez resucitada por Silva, los demás continuadores estarán obsesionados por el personaje. Al acabar con ella definitivamente Gaspar Gómez, ya analizaremos la razón, forzó a quienes lo siguieron tuvieran que aguzar el ingenio, con más bien poca fortuna, salvo Sebastián Fernández, para mantener en sus obras una relación lo más directa posible, sobre todo, con la vieja alcahueta. Así, Sancho de Muñón, en su Tragicomedia de Lisandro y Roselia, dedica un amplio espacio a desmontar el artificio de Silva [III, 1, 32 y SS.], pero, como Gómez de Toledo ha acabado con la vida de Areúsa y de Celestina, utiliza a Elicia como tercera en su obra, personaje que sistemáticamente en Celestina ha expresado su desdén por el oficio de alcahueta, lo que le obliga a hacer, incluso, trastocando el original, constantes alusiones a la relación entre Elicia y Celestina, para con ello seguir concentrando la atención en la vieja del original (Esteban Martín 1988).

El siguiente continuador, Sebastián Fernández, dado que Muñón acaba con Elicia, se encuentra sin ningún personaje estrechamente relacionado con Celestina para ejercer la alcahuetería. Sin embargo, en lugar de crear una nueva alcahueta, Sebastián Fernández recurre a Claudina (Esteban Martín 2000), la maestra continuamente recordada por Celestina y madre de Pármeno, lo que, además de la originalidad en la forma de entroncar con la obra modelo con respecto a sus antecesores, ya que supone la recreación en la Tragedia Policiana, partiendo de los datos del texto modelo, de un tiempo anterior al texto rojano, una precuela, por tanto, supone también reforzar y justificar la constante alusión a Celestina (Esteban Martín 1989a). Por último, en la Comedia Florinea, de Joan Fernández, y en la Comedia Selvagia, de Alonso de Villegas Selvago, Marcelia y Dolosina, alcahuetas de estas dos obras, 
respectivamente, constantemente recordarán episodios relacionados con la vieja Celestina (Esteban Martín 1989b), aunque ahora más desdibujados y en ocasiones forzados.

A todo lo anterior habría que añadir, como he señalado en otros trabajos, que la mayor parte de los ecos y reminiscencias fraseológicas que aparecen en estas continuaciones están relacionadas con parlamentos de Celestina. Así pues, la actitud de Silva refleja un dato fundamental: la obsesión que todos los continuadores tendrán por Celestina, obsesión que demuestra que todos se percataron de que era este el personaje más característico y llamativo de Celestina.

Dicho esto, Silva utiliza un recurso típico de las novelas de caballerías, la falsa muerte, que, además, era más que conocido y del agrado del público, de manera que construye un argumento que justifica que, en realidad, Sempronio y Pármeno creyeron que habían matado a la vieja, pero esta logró sobrevivir y, una vez recuperada, aquí está de nuevo lista para mediar en unos nuevos amores, en este caso los de Felides y Polandria. Pero conviene hacer hincapié en un rasgo esencial de la vieja Celestina en la obra de Silva: el aprendizaje del pasado, como ha comentado Consolación Baranda (Silva 1988: 53-57), y que será una de las mayores deficiencias en el tratamiento que de Celestina hará Gaspar Gómez de Toledo.

Pero Silva no solo recupera para el ciclo a la alcahueta, sino que, sin duda por herencia de la práctica en los libros de caballerías, deja la obra abierta para posibles continuaciones, no solo porque la vieja Celestina no morirá, sino porque la obra queda pendiente de que el matrimonio secreto entre Felides y Polandria se haga público y además dejará una serie de episodios inconclusos para posibles continuaciones. Y es aquí donde Gaspar Gómez de Toledo entra como continuador de un ciclo que sabe de éxito entre los lectores y que le llevará a que en su Tercera parte de la tragicomedia de Celestina no solo haga constantes referencias a Celestina, si bien, distintamente a Silva, sean referencias funcionales para establecer puentes entre su obra y la original (Esteban Martín 1987), sino fundamentalmente a la Segunda Comedia de Celestina.

En el título de la obra ya deja Gaspar Gómez de Toledo explícita su actitud de continuar la obra de Silva y de ahí que leamos "Tercera parte de la Tragicomedia de Celestina. Va prosiguiendo en los amores de Felides y Polandria, conclúyense sus desseados desposorios y la muerte y desdichado fin que ella ouo» ${ }^{6}$. En el extenso prólogo, Gaspar Gómez de Toledo vuelve a insistir en la relación de su obra con la de Silva, señalando de su obra que "va tan tosca en sus dichos quan subtil en las sentencias subtilíssimas la pasada, que es la donde ésta depende» (pág. 75), para más adelante instar a que debe ser leída la Segunda Celestina previamente: "Agora no me falta, después de tener la merced concedida de vuestra merced, sino rogar al lector que ésta leyere lea primero la Segunda, que es antes 
de ésta [...] Y ansí, porque el vulgo note la historia de do procede, suplico a vuestra merced se lo encargue» (pág. 77).

Tras esto comienza el texto de Gaspar Gómez de Toledo justo al día siguiente de que en la Segunda Celestina Felides y Polandria, una vez que se han desposado en secreto (cena XXXI) y han gozado de su amor (cena XL), queden a la espera de celebrar el matrimonio público. No voy a desarrollar aquí el amplio despliegue de menciones que a la Segunda Celestina se hacen en la obra de Gaspar Gómez de Toledo (Esteban Martín 1986: 129-153), pero sí me detendré en dos aspectos interrelacionados y que son esenciales para entender la actitud de Gaspar Gómez de Toledo: el cierre de los episodios que Silva dejó abiertos y la muerte de Celestina, porque ambas cuestiones son las que nos dan idea de la actitud de Gaspar Gómez de Toledo como continuador.

Lector atento de la Segunda Celestina, como no podía ser de otro modo, Gaspar Gómez de Toledo detecta las posibilidades dramáticas que tienen esos episodios inconclusos para un plan que se anuncia desde el mismo título de su obra, la tragedia final, de ahí que no continúe con la titulación de Silva de comedia y disponga tragicomedia.

En la cena XXXIX de la Segunda Celestina el fanfarrón Pandulfo demanda a Celestina diez doblas para armas argumentando que las suyas las había perdido en un lance cuando iba con Felides, lo cual es falso. Celestina, aprendiendo de lo que le ocurrió por no partir sus ganancias con Sempronio y Pármeno en Celestina (Silva: 555), le franquea la puerta, pero niega haber recibido cien doblas de Felides y, con astucia, le propone al fanfarrón ir a casa de Felides para comprobarlo (pág. 559), lo que es de todo punto inviable pues Pandulfo ha mentido en cuanto al lance que ha ocurrido acompañando a Felides. Ante esto, Pandulfo resuelve la situación diciendo que todo había sido una burla suya para saber si la vieja había recibido dinero de Felides (pág. 560). Aquí deja Silva este episodio que se retomará en la Tercera Celestina, en el auto II, cuando Sigeril aluda a esas cien doblas entregadas por Felides (Gómez de Toledo: 90). Más tarde, la vieja se jactará del engaño que hizo sobre Pandulfo (pág. 161), que, enterado, con la ayuda de Rodancho castigará a la vieja en este mismo auto, quedando cerrado el episodio.

El siguiente suceso que cerrará Gaspar Gómez de Toledo viene de la cena XXXIV de la Segunda Celestina en donde la vieja alcahueta aconseja a Elicia que reciba como amante a Barrada. Pese a las reticencias de Elicia, quien está con Albacín, Celestina decide cobrar cuatro ducados a Barrada como señal (Silva: 492), aunque Elicia lo seguirá rechazando. En el auto XXV de la Tercera Celestina Elicia y Albacín dialogan sobre la actitud de Celestina y su deseo de que Elicia acepte a Barrada, lo que desencadena la ira de éste, quien en el acto XXVI agredirá a la vieja y le robará cincuenta ducados. 
El tercer episodio está en relación con el anterior y supone un paso más en el desarrollo dramático que pone en marcha Gaspar Gómez de Toledo. En el auto XXXVII Elicia vuelve a comentarle a Albacín la insistencia de Celestina en que acepte a Barrada, lo que acentúan los deseos de venganza de Albacín que esta vez acuchillará en la cara a la vieja y se llevará a Elicia, quien no ocultará su odio hacia Celestina (Gómez de Toledo: 317), lo que supone entroncar al personaje de Elicia con las reticencias hacia la prostitución que tenía en Celestina al tiempo que elimina la posibilidad de que Elicia pudiese convertirse en una alcahueta en futuras continuaciones. Y buena prueba de ello es la debilidad de la configuración de Elicia como alcahueta en la obra de Sancho de Muñón.

Finalmente, en la Segunda Celestina se había aludido en diversos momentos a la relación entre Areúsa y Grajales (cenas XXIX y XXXIV), pero en la cena XXXV surgen algunos conflictos debido a los celos de Centurio, que, no obstante, no amedrentan a Grajales, a pesar de las fanfarronadas de aquel y sus compañeros (cena XXXVII). En el auto XIII de la Tercera Celestina, Areúsa visita a Elicia y le dice que ha de contarle «mil pasatiempos que con Grajales tengo» (pág. 154). Pero no será hasta el auto XXVII cuando se vuelva sobre este episodio, cuando Grajales, al ir a visitar a Elicia, se encuentra con Brauonel, quien mostrará su deseo por Areúsa: «No creo en quien me vistió si no voy más picado de Areúsa que muerto de hambre» (pág. 259). Tras los consejos de Celestina, Areúsa acepta como amante a Brauonel (pág. 277) y cuando están gozando de su relación son descubiertos por Recuaxo (auto XXXV), que se lo dirá a Grajales y este acabará con la vida de Areúsa (auto XL).

Como vemos, desde que Gaspar Gómez de Toledo decide continuar la obra de Silva parece planear en su mente la idea de acabar con todos los personajes que pudieran dar lugar a una posible continuación de su obra. Los tres episodios aquí expuestos suponen sendos castigos para Celestina que bien hubieran podido dar fin a la vida del personaje y de ahí que la muerte final resulte absurda para Heugas (1973: 55), pero en realidad forman parte del plan preconcebido por Gaspar Gómez de Toledo. Por un lado, desarrollar lo más posible el personaje de la vieja alcahueta y mantenerla hasta que se acabe con su más directa discípula, Areúsa. Por otro lado, intensificar la codicia de la vieja, uno de los rasgos más significativos del modelo original.

Llegados a este punto, Gaspar Gómez de Toledo ha ido cerrando episodios de la obra de Silva y su planteamiento requiere de la muerte de Celestina, que se produce al caerse por la escalera de su casa cuando, tras haberse celebrado el desposorio público de Felides y Polandria, pretende obtener un nuevo beneficio no solo de Felides, sino también de Polandria (Gómez de Toledo: 378), intensificando de este modo uno de los rasgos esenciales de Celestina, la codicia, como he señalado anteriormente. 
¿Qué lleva a Gaspar Gómez de Toledo a plantear un final de su obra de este modo? Pudiera interpretarse que nuestro autor quiere entroncar con el planteamiento trágico de la obra modelo; sin embargo, en mi opinión, esta interpretación chocaría con el deseo manifiesto de Gaspar Gómez de Toledo de continuar la obra de Silva, cuya obra sigue, pero no el espíritu de Feliciano de Silva de dejar abierta la obra para posibles continuadores. Así pues, me parece que la explicación al planteamiento de Gaspar Gómez de Toledo hay que buscarla en un deseo de alcanzar la fama entre unos lectores que ya habían manifestado el gusto por las obras anteriores y muy especialmente por las andanzas de la vieja Celestina, personaje más original que la historia de los amantes en cuyos amoríos media. Historias de amor había muchas, alcahuetas como Celestina ninguna.

Lo que planteo en este trabajo no es la búsqueda de la fama desde una perspectiva de tópico o tema, como tan bien analizó Lida de Malkiel (1983), sino de la fama popular, de gozar del éxito entre un público amplio y que es un rasgo muy significativo del Renacimiento, ligado, como no podía ser de otro modo, a la conciencia de autor que se desarrolla con respecto a la Edad Media. Como bien señala Jorge Roaro:

\begin{abstract}
para los sabios medievales, e incluso para los primeros humanistas, tanto el honor como la fama no eran sino manifestaciones de vanidad y de orgullo fatuo, completamente contrarias a la humildad cristiana; por lo tanto, es interesante cómo ambas cualidades fueron ganando reconocimiento mientras fue madurando el Renacimiento, hasta convertirse en cualidades protagónicas en la vida social del siglo XVI en adelante. (Roaro 2017: 48)
\end{abstract}

Cierto es que apuntes de este concepto de fama ya había en épocas anteriores, incluso Cicerón, en las Disputaciones Tusculanas, señalaba: «El honor alimenta las artes, y la gloria inflama a todos al cultivo de las mismas» (Roaro, 2017: 7), y, como vemos, lo ligaba especialmente a las artes, tal y como lo hará, en 1511, Erasmo de Rotterdam en su Elogio de la locura: «¿Y qué se podrá decir de los cultivadores de las artes? A todos ellos les es tan peculiar el Amor Propio, que sería más fácil de encontrar quien renunciase a la herencia paterna que a la fama de talento, sobre todo entre los actores, cantores, oradores y poetas, entre los cuales cuanto más ignorante es cada cual, tanto más se complace arrogantemente en sí mismo y se pavonea y se exalta más» (1953: 82), pero será con el Humanismo cuando ese deseo de fama se extienda hasta el punto de poderse hablar de una auténtica modernidad, como ha apuntado Garrote Pérez, que, en referencia al Lazarillo, dice: "la obra que redacta en su intimidad la saca a la consideración de todos, para buscar en el nivel de lo público un "mérito" ante los demás, el que, tras la lectura, la elogien por ser una obra bien hecha. 
Esta es la fama humanista, producto del esfuerzo y del trabajo de cada uno y, además, reconocido por todos en el nivel de lo público» (2004: 4).

Este gusto por la fama, centrándonos en los escritores, va ligado a la invención de la imprenta y también a factores de índole económica, como ha señalado González-Barrera: «la invención de la imprenta, el boom económico de las ciudades y la aparición de un mercado más o menos estable de lectores posibilitó que se creara en Europa una industria floreciente en torno a los libros y, que por primera vez, surgiera el factor económico como algo determinante para orientar la trayectoria editorial de los autores, que comenzaron a ver la literatura como una fuente de ingresos» (2015: s.p.).

En este contexto, considero que es este deseo de fama y de posteridad $y$, por qué no, de obtener unos ingresos económicos, el que hace que Gaspar Gómez de Toledo considere que cerrando un ciclo gozaría cuanto menos de la misma gloria que sus antecesores, al tiempo que cerraba la puerta a posibles continuaciones al haber acabado tanto con Celestina como con su más directa discípula, Areúsa. En otras palabras, creo firmemente que Gaspar Gómez de Toledo considera que al cerrar el ciclo alcanzaría tanta popularidad como Rojas y Silva.

La crítica moderna no ha tratado bien a Gaspar Gómez de Toledo. Ya he señalado al inicio de estas páginas cómo Urquijo se limita a definir a nuestro autor como "oscuro escritor toledano", la insigne Lida de Malkiel (1970) en su magistral estudio sobre Celestina solo alude en tres ocasiones a la Tercera parte de la tragicomedia de Celestina, señalando que no ha podido consultar dicha obra, tampoco le dedica mucha atención Heugas (1973) en su trabajo y en la actualidad solo contamos con la edición de Barrick (1973), de escasa difusión entre el público general, pese a que, amén de Celestina, sí que contemos con ediciones accesibles de la Segunda Comedia de Celestina, de la ya mencionada Consolación Baranda (1988), e incluso de la Tragicomedia de Lisandro y Roselia a cargo de mi colega Rosa Navarro Durán (2009), por lo que me parece más que interesante que se abordase no solo una edición de esta obra, sino entrar en el estudio de la repercusión de Gaspar Gómez de Toledo en los autores posteriores, amén de poner en valor los aciertos dramáticos y estilísticos que la obra tiene. 


\section{Bibliografía:}

CheValier, Maxime (1976), Lectura y lectores en la España del siglo XVI y XVII, Madrid, Ediciones Turner.

Esteban MARTín, Luis M. (1986), "La presencia de La Celestina en la Tercera Celestina de Gaspar Gómez de Toledo», Tesina de Licenciatura, Universidad Complutense, Madrid.

- (1987), "Huellas de La Celestina en la Tercera Celestina de Gaspar Gómez de Toledo", Celestinesca, 11, 2, pp. 3-19.

- (1988), "Huellas de Celestina en la Tragicomedia de Lisandro y Roselia, de Sancho de Muñón», Celestinesca, 12, 2, pp. 17-32.

-. (1989a), "Huellas de Celestina en la Tragedia Policiana, de Sebastián Fernández", Celestinesca, 13,1, pp. 31-41.

- (1989b), "Huellas de Celestina en la Comedia Florinea y en la Comedia Selvagia», Celestinesca,13,2, pp. 29-38.

- (1991-92), "Feliciano de Silva en el ciclo celestinesco», La Corónica, pp. 42-49.

- (2000), "Claudina, del recuerdo a la vida», Celestinesca, 24, 1-2, pp. 77-86.

- (2003), "La carta amorosa en la trama de la descendencia directa de Celestina», LEMIR, 7, s.p.

García Galiano, Ángel (1992), La imitación poética en el Renacimiento, Deusto, Publicaciones de la Universidad de Deusto. Edition ReichenbergerKassel.

GARrote Pérez, Francisco (2004), "Interpretación de La vida del Lazarillo de Tormes y de sus fortunas y adversidades», en VI Reunión Científica sobre Humanistas Españoles, León y San Pedro de Dueñas, 2004, pp. 1-20.

Genette, Gerard (1989), Palimpsestos: La literatura en segundo grado, trad. de Ceñia Fernández Prieto, Madrid, Taurus, 1989.

Gómez de Toledo, Gaspar (1973), Tercera parte de la tragicomedia de Celestina, ed. Mac E. Barrick, Philadelphia, University of Pennsylvania Press. GonZÁlez-BARRERA, Julián (2015), "Hacia una cartografía de la fama: el caso de Lope de Vega", en Tiempo e historia en el teatro del Siglo de Oro, Actas selectas del XVI Congreso Internacional [en línea], Aix-en-Provence, Press universitaires de Provence. <http:/books.openedititon.org/ pup/4724>.

Heugas, Pierre (1973), La Célestine et sa descendance directe, Bordeaux, Ed. Bière.

KirbY, Steven D. (1989), "¿Cuándo empezó a conocerse la obra de

Fernando de Rojas como Celestina?», Celestinesca, 13, 1, pp. 59-62.

LÁzaro Carreter, Fernando (1978), "Lazarillo de Tormes» en la picaresca, Barcelona, Ariel. 
LÁZARo Carreter, Fernando (1976), «Sobre el género literario», en Estudios de poética (la obra en sí), Madrid, Taurus, pp. 113-120.

Lida de Malkiel, María Rosa (1970), La originalidad artística de "La Celestina", Buenos Aires, Eudeba.

- (1983), La idea de la fama en la Edad Media castellana, Madrid, FCE.

Muñón, Sancho de (1872), Tragicomedia de Lísandro y Roselia, Llamada Elicia, y por otro nombre cuarta obra y tercera Celestina, Madrid, Imprenta y Estereotípia de M. Rivadeneyra (colección de libros españoles raros o curiosos) III.

Norton, F.J. (1966), Printing in Spain 1501-1520, with a note on the early editions of "La Celestina", Cambridge, University Press.

Pérez-Rioja, José Antonio (1988), La creación literaria, Madrid, Ed. Tecnos. RoARO, Jorge (2017), «El Humanismo renacentista español de los siglos XV y xVI, visto a través de la Retórica, la reflexión filosófica y la búsqueda de la virtud», Tesis doctoral, Salamanca, Universidad de Salamanca.

Rotterdam, Erasmo de (1953), Elogio de la locura, Madrid, Espasa Calpe.

Segundas Celestinas (2016), ed. de Rosa Navarro Durán, Madrid, Fundación José Antonio Castro.

Serrano Sanz, Manuel (1902), "Noticias biográficas de Fernando de Rojas autor de La Celestina, y del impresor Juan de Lucena», Revista de Bibliotecas, Archivos y Museos, VI, pp. 24-299.

Silva, Feliciano de (1988), Segunda Celestina, ed. Consolación Baranda, Madrid, Cátedra.

Ticknor, M.G. (1851), Historia de la literatura española, trad. de Pascual de Gayangos y Enrique de Vedia, I, Madrid, Imprenta M. Rivadeneira.

Urouijo, J. de (1910), "La tercera Celestina y el canto de Lelo", Revue Internationale des Études Basques, pp. 573-586.

Villegas, Alonso de (1554), Comedia Selvagia, Toledo. 
